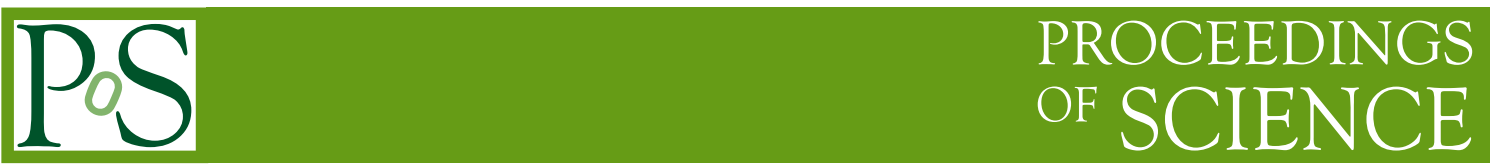

\title{
SQUID-based beam position monitor
}

\author{
Selçuk Hacıömeroğlu* \\ IBS, Center for Axion and Precision Physics, South Korea \\ Email:selcuk@ibs.re.kr
}

\section{David Kawall}

University of Massachusetts Amherst, Physics Department, USA

Email:kawall@physics.umass.edu

\section{Yong-Ho Lee}

KRISS, Center for Biosignals, South Korea

Email:yhlee@kriss.re.kr

\section{Andrei Matlashov}

IBS, Center for Axion and Precision Physics, South Korea

Email : andrei@ibs.re.kr

\section{Zhanibek Omarov}

IBS, Center for Axion and Precision Physics, South Korea

KAIST, Department of Physics, South Korea

Email:zhanik@kaist.ac.kr

\section{Yannis K. Semertzidis}

IBS, Center for Axion and Precision Physics, South Korea

KAIST, Department of Physics, South Korea

Email: yannis@bnl.gov

The proton electric dipole moment (pEDM) experiment is proposed to be done with two counterrotating beams in an all-electric storage ring. Radial magnetic field is one of the most critical systematic errors in the experiment as it mimics the EDM signal. Its secondary effect is a vertical split between the counter-rotating beams. According to the proposal of the pEDM experiment, that split should be monitored and compensated to picometer level by the beam position monitoring systems (BPM). We propose a BPM using Superconducting QUantum Interference Devices or SQUIDs to measure the beam splitting with such resolution that corresponds to magnetic field measurement with $10^{-18} \mathrm{~T}$ (aT) resolution. In this paper we experimentally demonstrate that the white noise of such magnetometers and SQUID read-out electronics can be decreased by more than two orders of magnitude down to $25 \mathrm{aT} / \sqrt{\mathrm{Hz}}$ by using 5 hour signal averaging. It demonstrates very high long-time stability and low intrinsic fluctuation level in the instrument. We expect that our next BPM version will be able to reach $<2 \mathrm{aT} / \sqrt{\mathrm{Hz}}$ white noise level after 5 hour averaging. This noise level can be further decreased by using recently designed single-chip magnetometers with sub-micron size Josephson junctions.

The 39th International Conference on High Energy Physics (ICHEP2018)

4-11 July, 2018

Seoul, Korea

${ }^{*}$ Speaker. 


\section{Introduction}

The pEDM experiment aims to search for the electric dipole moment (EDM) of the proton with $10^{-29} e \cdot \mathrm{cm}$ sensitivity. The experiment proposes to apply electric field on the counter-rotating beams perpendicular to their initial spin in an all-electric storage ring, and then measure the spin precession rate on the vertical plane. The details of the proposed experiment can be found at $[1,2]$. For a particle at rest, the electric $(\vec{E})$ and magnetic $(\vec{B})$ fields precess the spin the same way as they couple with $\operatorname{EDM}(\vec{d})$ and $\operatorname{MDM}(\vec{\mu}): \vec{\omega}=\vec{d} \times \vec{E}+\vec{\mu} \times \vec{B}$.

For a particle in a storage ring, this equation is modified by relativistic terms and the Thomas spin precession term to give the so-called T-BMT equation $[3,4]$. Then, the radial component of the spin precession rate $\left(\omega_{r}\right)$ is several nrad/s for radial electric field $E_{r}=10^{7} \mathrm{~V} / \mathrm{m}$ and $|\vec{d}|=$ $10^{-29} e \cdot \mathrm{cm}$. Comparing this with the MDM-related terms shows that the net radial magnetic field $\left(B_{r}\right)$ should be kept at attoTesla level for the above-mentioned conditions. This is clearly impossible with a magnetic shielding alone [5], requiring continuous measurement and active compensation.

\section{Measuring the average radial magnetic field}

The average radial magnetic field can be measured by means of beam dynamics of the counterrotating beams in the storage ring. It can be expressed as a function of the azimuth $\theta$ as

$$
B_{r}(\theta)=\sum_{N=0}^{\infty} B_{r N} \cos \left(N \theta+\phi_{N}\right)
$$

where $B_{r N}$ and $\phi_{N}$ are the radial magnetic field strength and the phase for the $N^{\text {th }}$ multipole.

Besides making spin precession, $B_{r}$ also splits the counter rotating beams by the Lorentz force (See Figure 1). Its equation of motion on the vertical plane is

$$
\frac{d^{2} y}{d \theta^{2}}+Q_{y}^{2} y=\frac{\beta c R_{0}}{E_{r}} \sum_{N=0}^{\infty} B_{r N} \cos \left(N \theta+\phi_{N}\right)
$$

where $Q_{y}$ is the vertical tune, $R_{0}$ is the radius of the ring, $\beta$ and $c$ are the relativistic speed and the speed of light respectively. Its solution is

$$
\delta y(\theta)=y_{0} \cos \left(Q_{y} \theta+\phi_{Q}\right) \pm \sum_{N=0}^{\infty} \frac{\beta c R_{0} B_{r N}}{E_{r}}\left[\frac{1}{Q_{y}^{2}-N^{2}}\right] \cos \left(N \theta+\phi_{N}\right),
$$

with the first term corresponding to the betatron oscillation. The terms $N>0$ average out to zero over the storage. Therefore, assuming a smooth beta function, they don't have a net contribution to the vertical offset $\delta y$. Then, each beam gets an average offset of

$$
\delta y= \pm \frac{\beta c R_{0} B_{r} 0}{E_{r} Q_{y}^{2}} .
$$

For $B_{r 0}=10$ aT and the proposed ring with $\beta=0.6, R_{0} \approx 80 \mathrm{~m}, Q_{y}=0.4$ and $\left\langle E_{r}\right\rangle=5 \mathrm{MV} / \mathrm{m}$, the counter-rotating beams will have a net split of $\Delta y=2|\delta y| \approx 0.4 \mathrm{pm}$.

The vertically separated beams induce a net magnetic field $\left(B_{x}\right)$ on the horizontal plane. It can be measured by a magnetic field sensor located several cm away horizontally with the measurement 


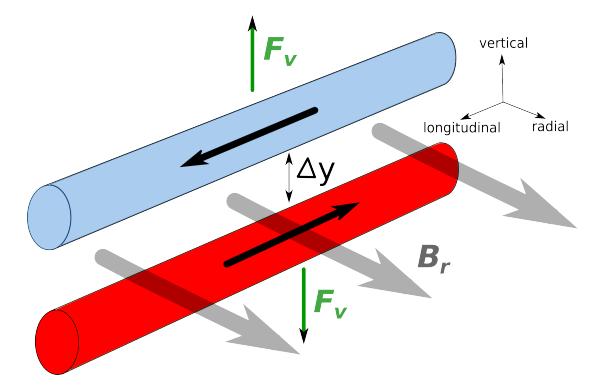

Figure 1: The counter rotating beams are split by $\Delta y$ due to the radial magnetic field $B_{r}$.

being proportional to the separation $\Delta y$. Assuming $10 \mathrm{~mA} \mathrm{DC}$ current for beams and the conditions given above, the total field at $x=2 \mathrm{~cm}$ away from the beams becomes $B_{x}=4 \mathrm{aT}$.

Typically, sub-fT level measurement is shown to be achievable within $100 \mathrm{kHz}$ bandwidth by using DC SQUID magnetometers/gradiometers [6]. This allows measurement of an aT level field by time averaging. Considering that it is sensitive to only alternating field, one needs to pick an operation frequency $f_{m}$ for the SQUID device.

By changing the strength of the quadrupoles, one can modulate the vertical tune at the operation frequency of the SQUIDs as

$$
Q_{y}(t)=Q_{y 0}\left[1-A \cos \left(\omega_{m} t\right)\right]
$$

with $\omega_{m}=2 \pi f_{m}$ and the amplitude of modulation $A$. Using the approximation $\left(1-A \cos \left(\omega_{m} t\right)\right)^{-2} \approx$ $1+2 A \cos \left(\omega_{m} t\right)$ for a small $A$ and substituting it into Equation 2.5, $B_{x}$ at the modulation frequency $\omega_{m}$ becomes

$$
B_{x}=\frac{\mu_{0} I \Delta y}{\pi r^{2}} 2 A \cos \left(\omega_{m} t\right) .
$$

Putting $I=10 \mathrm{~mA}, \Delta y=0.5 \mathrm{pm}, A=0.1$ and $r=2 \mathrm{~cm}$ into Eq 2.6 gives $B_{x} \approx 1 \mathrm{aT} \cos \left(\omega_{m} t\right)$.

As a reference, with an array of 8 SQUIDs of $0.5 \times 10^{-15} \mathrm{~T} / \sqrt{\mathrm{Hz}}$ sensitivity at $1 \mathrm{~Hz}$ bandwidth $(0.5 \mathrm{fT} / \sqrt{\mathrm{Hz}})$, it requires $2 \times 10^{4}$ seconds of averaging to achieve $\mathrm{SNR} \approx 1$ as $B=0.5 \times$ $10^{-15} \mathrm{fT} / \sqrt{8 \times 2 \times 10^{4}}=1.25 \mathrm{aT}$.

\section{Preliminary tests with the SQUID-based BPM}

SQUID-based magnetometers can measure magnetic field variations with unprecedented noise level below $1 \mathrm{fT} / \sqrt{\mathrm{Hz}}$. This is why they became the best candidates for the beam position monitors in the pEDM experiment. In addition to high resolution, the SQUID-based magnetometers have sufficient bandwidth and compact size that allows using multi sensor arrays placed along the beam trajectory inside a superconductive shielding structure.

Figure 2 shows the 3D drawing of the BPM, designed at KRISS. It will operate in vacuum at 4 Kelvin. The white noise of such magnetometers is approximately $0.5 \mathrm{fT} / \sqrt{\mathrm{Hz}}$. They are positioned on the horizontal plane to measure the vertical split.

The BPM works inside a magnetically shielded room (MSR). The data transfer between the SQUIDs and the computer is done via fiber lines to minimize electromagnetic noise. 


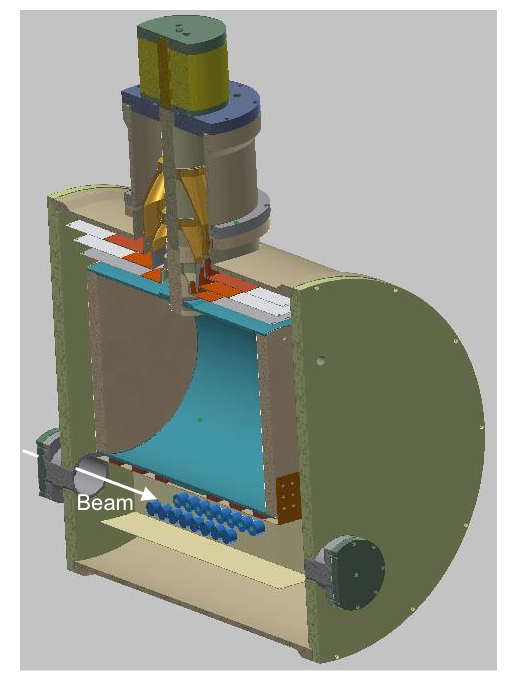

Figure 2: The beam (white arrow) will pass between two arrays of SQUID gradiometers. The SQUID sensor and the pickup loops (dark blue) will be kept cold to LHe level. Above the SQUIDs is the LHe tank, shown with turquoise layer. The whole setup can fit in a cube of $1 \mathrm{~m}^{3}$ volume.

The operation frequency was chosen to be around 1-10 kHz for minimizing the external noise. This will be achieved by modulating the vertical tune $Q_{y}$ as mentioned above.

We conducted preliminary tests with a setup having a similar SQUID electronics but a different design of pickup loops and dewar. The dewar and the eight SQUID gradiometers are shown in Figure 3. This system was also originally designed at KRISS for biomagnetic applications. It has 8 axial wire-wound first-order gradiometers positioned along a bottom line inside a fiberglass dewar. Each gradiometer has $20 \mathrm{~mm}$ diameter and $50 \mathrm{~mm}$ baseline and bonded to the double relaxation oscillation (DROS) SQUID current sensor. DROS SQUIDs have a large flux-to-voltage transfer coefficient that minimizes the contribution of the direct read-out electronics noise. The white noise of such gradiometers is about $3 \mathrm{fT} / \sqrt{\mathrm{Hz}}$ at frequencies above $1 \mathrm{~Hz}$.

For the long-term measurements, the magnetic field was induced by two parallel traces with $100 \mu \mathrm{m}$ separation and carrying opposite currents of $100 \mu \mathrm{A}$ on a PCB. We applied a sinusoidal current of $300 \mathrm{~Hz}$, corresponding to around $200 \mathrm{fT}$ field at the bottom of the pickup loop.

The measurements showed more than two orders of magnitude suppression of white noise from the gradiometers and the SQUID read-out electronics (Figure 4) after 5-hour averaging, corresponding to $25 \mathrm{aT} / \sqrt{\mathrm{Hz}}$. This indicates very high time stability and low intrinsic fluctuation level in the instrument, including cryogenics and semiconductors, both analog and digital electronics.

The prototype (Figure 2) includes 16 magnetometers with two-turn $17 \mathrm{~mm}$ diameter pick-up coils bonded to DROS SQUIDs. It allows us to expect more than 3 times lower white noise floor, i.e. about $8 \mathrm{aT} / \sqrt{\mathrm{Hz}}$, after 5 hour averaging. For the further noise decrease we expect using singlechip integrated magnetometers similar to ML12 reported in [6] but with chip size $24 \times 24 \mathrm{~mm}^{2}$. Such magnetometers have white noise below $0.2 \mathrm{fT} / \sqrt{\mathrm{Hz}}$ at frequency above $1 \mathrm{kHz}$. Using this prototype, we are expecting to measure the relative beam position with a $1 \mathrm{~nm} / \sqrt{\mathrm{Hz}}$ (corresponding to $\approx 2.5 \mathrm{aT} / \sqrt{\mathrm{Hz}}$ ) sensitiviy at 5-hour averaging. 


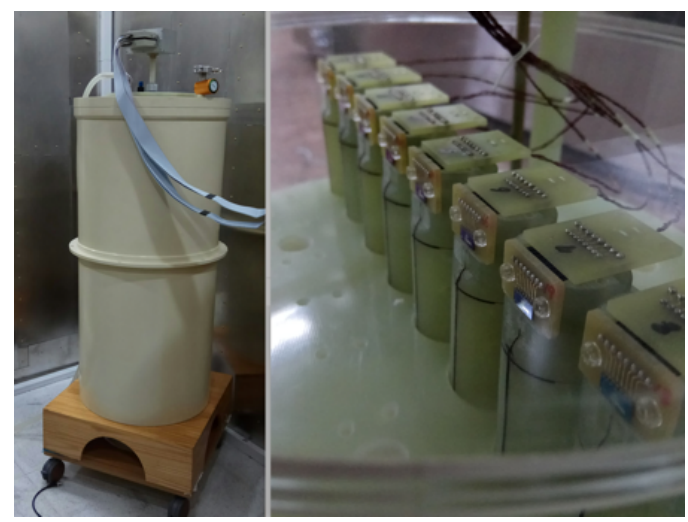

Figure 3: Time-averaging measurements were done with a setup having the same electronics but a different dewar (Left) and gradiometer (Right) designs.

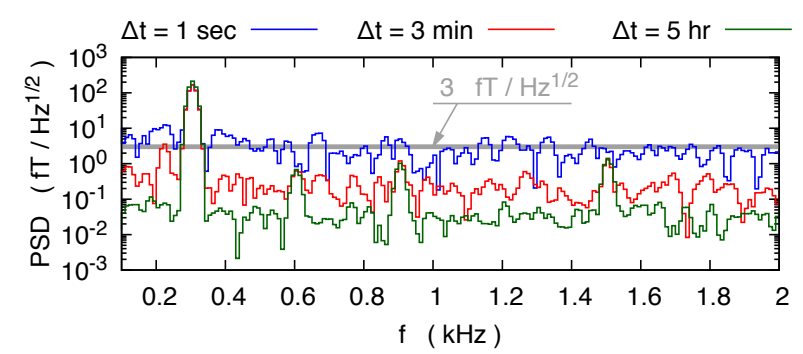

Figure 4: $100 \mu \mathrm{A}$ AC current was applied through the parallel traces on a PCB board, inducing $200 \mathrm{fT}$ RMS field on the pickup. The noise averaged in $1 \mathrm{~s}$ is a few $\mathrm{fT}$, consistent with the $3 \mathrm{fT} / \sqrt{\mathrm{Hz}}$ sensitivity of the SQUIDs. The noise decreases down to $25 \mathrm{aT} / \sqrt{\mathrm{Hz}}$ after 5 hours of averaging.

\section{Acknowledgement}

IBS-Korea (project system code: IBS-R017-D1-2018-a00) supported this project.

\section{References}

[1] F.J.M Farley et al., New Method of Measuring Electric Dipole Moments in Storage Rings, Physical Review Letters 93, (2004) 052001.

[2] V. Anastassopoulos et al., [EDM Collaboration], A storage ring experiment to detect a proton electric dipole moment, Review of Scientific Instruments 87,(2016) 115116.

[3] V. Bergmann, L. Michel and V.L. Telegdi, Physical Review Letters 2, Precession of the Polarization of Particles Moving in a Homogeneous Electromagnetic Field, (1959) 435.

[4] T. Fukuyama and A.J. Silenko, Derivation of T-BMT equation for a particle with electric dipole moment, International Journal of Modern Physics A 28, 29, (2013) 1350147.

[5] I. Altarev et al., A large-scale magnetic shield with $10^{6}$ damping at millihertz frequencies, Journal of Applied Physics 117, (2015) 183903.

[6] M. Schmelz et al., "Field-stable SQUID magnetometer with subfT- $\sqrt{\mathrm{Hz}}$ resolution based on sub- $\mu$ m cross-type Josephson tunnel junctions", Supercond. Sci. Technol. 24, (2011) 065009(5pp). 